\section{REFERENCES}

Chang, J, J, On-line multidimensional scaling programs with interactive graphical displav. Behavior Research Methods \& Instrumentation, $1973,5,99-103$.

Coombs, C. A theory of data. New York: Wiley, 1964.

Daalder, H., \& Rusk, J. G. Perceptions of party in the Dutch parliament. In S. C. Patterson and John C. Wahlke (Eds.), Comparative legislative behavior. New York: Wiley, 1972. Pp. 143-198.

Guttman, L. A general nonmetric technique for finding smallest coordinate space for a configuration of points. Psychom etrika, $1968,33,469-506$

Kruskal, J. B. Multidimensional scaling by optimizing goodness of fit to a nonmetric hypothesis. Psychometrika, 1964, 29, $1-27$.

Rusk, J. G., \& Weisberg, H. F. Perceptions of presidential candidates: Implications for electoral change. Midwest Journal of Political Science, 1972, 16, 388-410.

Spence, I. A Monte Carlo evaluation of three nonmetric multidimensional scaling algorithms. Psychometrika, 1972. $37,461-486$

Weisberg, H. F., \& Rusk, J. G. Dimensions of candidate evaluation. American Political Science Review, 1970, 64, 1167-1185.

\section{NOTES}

1. Such analysis is reported in Weisberg and Rusk (1970) and Rusk and Weisberg (1972).

2. The formal scaling problem is elegantly stated by Coombs (1964).

3. Nonmetric multidimensional scaling is presented in Kruskal (1964) and Guttman (1968).

4. For example, see Spence (1972).

5. Daalder and Rusk (1972) provide an example of multidimensional scaling of party spaces in the 12-party Dutch setting.

6. The CENTER and NORMALIZE commands were issued after the INITIAL command to facilitate display of the configuration, but these have no effect on the scaling.

7 . When all correlations are positive, we have found that dropping the first general component y ields a superior starting configuration. IMDS has this capability but we have not used it in this illustrative session, since evaluations of the left- and right-wing parties are negatively correlated.

8. The terminal user cannot see the solutions of Figs. 6 and 9 simultaneously and, therefore, could not determine where the differences between them occur. Addition to the program of an algorithm to compare spatial configurations would solve this problem.

9. Normally the prompting employed with interactive programs must be terse. We have found, however, that this applies only to nongraphic terminals. The speed of graphics term inals eliminates the terseness requirement.

Behavior Research Methods \& Instrumentation

1974, Vol. 6, No. 2, 194-200

\title{
The Minnesota interactive statistical system
}

\author{
RONALD E. ANDERSON, JAMES CLEARY, JONATHON GROSS, \\ DON McTAVISH, RICHARD MELSON, and JOHN VINCENT \\ Department of Sociology, University of Minnesota, Minneapolis, Minnesota 55455
}

\begin{abstract}
The Minnesota Interactive Statistical System (MISS) is reviewed as a successful application of on-line minicomputing in an instructional and research context. The MISS system is described with regard to (1) the instructional and research needs which prompted its development, (2) its programming design, (3) its operating system, (4) its computer hardware requirements, and (5) the continued innovative minicomputing development that it has inspired.
\end{abstract}

\section{INTRODUCTION}

The Minnesota Interactive Statistical System (MISS) is an integrated system of statistical programs for the analysis of social science data through minicomputing. The system was developed by the Sociology Department of the University of Minnesota as an educational and research facility. It was designed to maximize both ease of use and flexibility of analysis, so that both the novice user and the experienced researcher would find interaction with MISS a rewarding experience. The MISS system has some general resemblance to a number of other interactive instructional systems, such as IMPRESS, ISIS, and SIPS (Anderson, 1971; Anderson \& Coover, 1972; Cline \& Meyers, 1970). It is particularly unique in being operable on a small minicomputer.

As an integrated and interactive system, MISS permits the analysis of a data set to be very flexible. The user is able to develop his statistical analysis in a stepwise manner, viewing the results of one statistical analysis subroutine before deciding which other analysis to request next; and the output of some analysis subroutines may be optionally used as input for others (for example, the correlation matrix generated from raw data input by subroutine CORR may be stored in memory of MISS for use in higher level correlational analyses). Meanwhile, the rapid feedback of the interactive system permits the user to maiñtain a close awareness of his data, while it speeds the learning process and makes the task more interesting.

The statistical analysis programs and the possible paths of sequential analysis are represented in the schematic overview of MISS in Fig. 1. As indicated by the diagram, data may be entered into MISS in any of three basic forms: as raw data, as a frequency distribution, or as a matrix (either a correlation matrix, a bivariate table matrix, or a factor matrix). The arrows leading from a data type to the blocks representing the statistical analysis subroutines indicate which programs are compatible with each of the data types. Thus, it is possible to analyze raw data (on more than one variable) with any program in the system, provided that the raw 


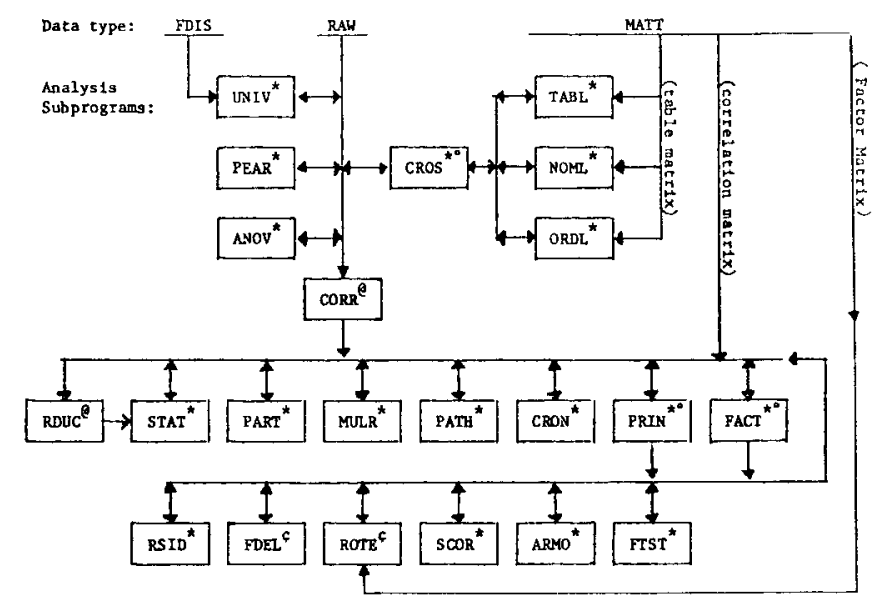

Fig. 1. Schematic overview of MISS. The arrows connecting the squares above generally indicate the alternative paths by which one's analysis might proceed given the type of data one has. The symbols associated with the program names are interpreted as follows: *-the program does not alter the data which is stored in memory of MISS; @ the program changes the form of the data in memory (i.e., from raw data into a correlation matrix); ${ }^{*}$-the program leaves the stored data unchanged but stores new output also; 1 -the program alters the stored factor matrix but not the correlation matrix. data are transformed properly before requesting those programs requiring such transformations. For example, one must request to have a correlation matrix computed from the raw data (by calling subprogram CORR) before requesting that factor analysis be performed (by calling PRIN), and that the latter be done (so that a factor matrix is computed and stored in memory) before requesting a factor rotation (by calling ROTE). One is considerably more limited, on the other hand, if his data are in the form of a frequency distribution. Then he may request only a univariate description of his data (by calling UNIV).

As can be inferred from the listing of analysis programs in Table 1, MISS permits univariate and bivariate statistical descriptions of data sets representing nominal-, ordinal-, and interval-level measurements, while its multivariate descriptions usually require interval-level data (with the exception of subprogram CROS, which has the capability of producing up to first-order partial tables for nominal- or ordinal-level data). This selection of statistical analysis subroutines has proven very useful in meeting our educational needs, as well as a broad spectrum of our research needs.

\section{THE INSTRUCTIONAL ENVIRONMENT}

The MISS system services a number of graduate and undergraduate instructional settings. Among them are (1) courses in the use of computers in the social sciences, (2) courses in statistics and research methodology, and (3) a variety of individual and faculty dissertations and projects. A brief description of the second usage will illustrate the breadth and character of the system's support of a viable instructional environment.
Our three-quarter undergraduate sequence in statistics and research methods aims at introducing the student to the design, analysis, procedures, and issues of inquiry. During the three quarters, small groups of students design, conduct, and analyze data for two or three studies of their choosing. The first of these calls for gathering 70-100 responses to a questionnaire of their design (enough to be statistically adequate and clearly show the summarizing utility of statistics and computers), getting these data into analyzable form on the MISS and/or batch-oriented systems, learning enough about each system to get started and to do univariate analysis of key questions-all within the first 2-3 weeks of the first quarter. This means that we need to quickly familiarize the students with these systems. The MISS system is very easy to learn, and it is structured in a way that permits us to show meaningful output with little effort. A simplistic but comprehensive user-oriented instructional manual (Cleary, 1973) facilitates this task.

A number of different data sets have also been prestored in the system for use in the statistics/methods sequence. Three recent data sets are (1) a study of juvenile offenders in group homes, conducted by a Minnesota state agency; (2) a 100-case subset of the NORC GENERAL social survey data; and (3) a 66-case subset of a national survey of dental students, conducted by John Vincent. " Code books of these projects are provided for student groups. ${ }^{2}$ Generally, the data stored in the MISS system are random samples from larger data sets. This raises issues of statistical inference, which are a useful focus for the inferential statistics course projects. The prestored sample data sets can easily be changed from quarter to quarter to avoid having to guard against 
hand-me-down projects.

These prestored data sets are used in three major ways. First, the data on file permit immediate use of the system on interesting and real data during labs. Second, since some student projects may not be adequate for some types of analysis, we can quickly shift assigned projects over to archived prestored data. Finally, these data sets permit an "interactive" shuttle between the sample and the full data set, using the sample as a "testing" ground for ideas which can then be worked out on the larger data set on the batch system. Thus, we have found the MISS system helps to keep the focus on the pursuit of inquiry, on comparative use of statistical tools in the context of a self-rescuing, learning-risk absorbing system.

\section{PROGRAMMING DESIGN}

The MISS system is implemented with the LIBRA time-sharing operating system and the FOCAL interpreter, an algebraic language similar to FORTRAN. The library commands of the operating system can be executed within the FOCAL programs. This permits extensive integration of the numerous subprograms comprising the MISS system.

The text which appears to the user, however, and which he uses to interact with the system is a natural language text. An illustration of the Teletype communication involved in performing a multiple-regression analysis is given in Fig. 2. After the first MISS program (the master drive program) is retrieved from disk memory by means of the FOCAL library call command and is executed with the FOCAL go command, the interaction proceeds in an English dialogue.

MISS begins by requesting the appropriate entry code, supplies recent system news (optional), asks whether the data are prestored in disk memory (DISK) or are to be entered via the Teletype (TTY), and asks what format characterizes the data (see Table 2). Following data entry, MISS presents the user with the data mode action commands (abbreviations of English commands) which may be used to edit the data in any of a variety of ways (see Table 2). From data mode the user may enter analysis mode to actually analyze the data in any of a number of ways (see Table 2). Since data mode and analysis mode can each be entered from the other, one can conveniently move between data manipulation and data analysis as often as the problem requires. And at any time the user may request new data under a similar or different format, or he may log off the system. Numerous error checks have been built into the system in anticipation of nearly any kind of user error (such as requesting an analysis program without the appropriate data type). This minimizes the likelihood of any serious user problems developing while using MISS.

One of the overriding considerations in past and

Table 1

Program

Name

UNIV Computes univariate descriptive statistics and optional frequency distribution from raw data. UNIV recognizes missing data and works on one variable or all of them.

PEAR Computes Pearson correlation and bivariate linear regression information. PEAR recognizes missing data.

ANOV Performs one-way or two-way analysis of variance.

CROS Generates bivariate and first-order partialled frequency distributions and percentages from raw data. CROS recognizes missing data.

TABL Generates a three-way percentage table for bivariate distributions which have been generated by CROS or input under MATT data format.

NOML Outputs several nominal-level table statistics for bivariate tables which have either been generated by CROS or input under MATT data format.

ORDL Outputs several ordinal-level table statistics for bivariate tables which have either been generated by CROS or input under MATT data format.

CORR Computes correlation and covariance matrices from raw data input and can store this output in memory for use in other programs.

RDUC* Allows the user to reduce the size of a correlation matrix which is stored in memory.

STAT* Informs the user of the state of the data in memory relevant to programs requiring correlation matrix and factor matrix input.

PART* Generates partial correlations up to the eight-order.

MULR* Computes multiple correlation and regression information.

PATH* Performs path analysis for nonrecursive path models.

CRON* Performs an item analysis and computes Cronbach's alpha as a reliability estimate.

PRIN* Performs principal axis factor analysis using either the common factor model or the principal components model.

FACT* Performs confirmatory factor analysis by extracting arbitrary orthogonal factors which are specified as hypothesis vectors by the user.

RSID $\dagger$ Generates the factor product matrix and the residual correlation matrix for factor matrices generated by either PRIN or FACT.

FDEL $\dagger$ Permits the deletion of factors from an unrotated factor matrix stored in memory.

ROTE $\dagger$ Enables the user to rotate according to either the varimax or quartimax criterion a factor matrix which has been computed by either PRIN or FACT.

SCOR $\dagger$ Computes factor scores for individual cases based on unrotated or rotated factor patterns generated by either PRIN or FACT.

ARMO† Computes Armor's theta as a reliability estimate for a scale based on principal components factor analysis.

FTST $\dagger$ Computes reliability and validity estimates for a scale based on the common factor analysis solution.

*The correlation matrix required as input by these programs may be either input directly under MATT data format or computed from raw data by CORR.

$\dagger$ These programs require that a factor matrix and a correlation matrix be stored in memory. 


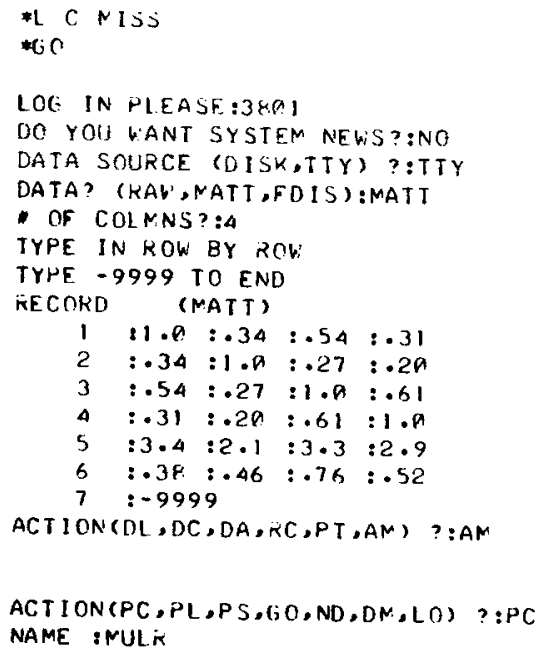

MULTIPLE REGRESSION

NUMBER OF CASES?:133

Fig. 2. A sample a nalysis using MISS.

future MISS development is the provision of sufficient data storage capacity for user analysis requirements. This has led to setting the maximum size of any raw data matrix at roughly 700 elements (for example, 70 cases on 10 variables) and the maximum size of any correlation matrix or bivariate table matrix at 10 or fewer columns and rows (a 10 by 10 matrix is the largest permissible). ${ }^{3}$ In general, this space limitation has not seriously interfered with the instructional goals of MISS, though in a few actual research cases it precluded the use of MISS in collapsing the raw data matrix. This suggests that interactive minicomputing may be better suited to the second-stage analyses of correlation matrices and bivariate tables than to the first-stage reduction of large data sets.

\section{OPERATING SYSTEM}

The standard LIBRA operating system has two features that make it particularly suitable for MISS: The system will time-share up to five users and it will maintain a library of programs. The library is accessed by the addition of a library command in FOCAL which allows programs to be saved and called from the disk. In the interest of efficiency, up to three users are kept in memory at the same time, reducing the number of disk swaps needed to service the users. The result is a very responsive system.

When LIBRA was first installed on our PDP.8/L and before MISS was conceived, the program library was quickly filled with stand-alone statistical programs. We were confronted with a number of problems. First, there was a trade-off between program size and variable storage, which greatly limited the scope of problems tackled. Second, much precious program space was used up by separate data entry and user interaction routines

Table 2

Descriptions of MISS Codes

Keyword Description

(1) Data Description Codes

DISK Data source located on disk.

TTY Data to be input from Teletype.

RAW Data organized as a MATRIX (two-dimensional) with columns designated variables and rows designated records (cases).

MATT (Matrix table) Data organized as a two-dimensional correlation or factor matrix or cross-tabulation table.

FDIS Data organized as a frequency distribution, with each record consisting of a score and its frequency.

(2) Data Mode Action Commands

DL (Data list) to type a printout of one's data between the limits specified; that is, one or more records are printed. This is most useful in detecting errors or in documenting the state of one's data.

DC (Data change) to correct, insert, or delete from an existing data set.

DA (Data append) to add data on to end of existing data set.

RC (Recode) allowing recoding of selected data values.

PT (Paper tape) to begin the punching of a paper tape of one's data set.

AM (Analysis mode) switches user from data mode to analy sis mode.

(3) Analysis Mode Action Commands

PC (Program call) to select a program and then to begin executing it.

PL (Program list) to list names of all available programs (analysis task modules).

PS (Program string) to request construction of a series of programs. This program string (series) can be used later on to initiate and automatically control the execution of a sequence of programs.

GO Go actually initiates the execution of a PS (program string), used after a PS action.

ND (New data) to enter additional data, destroying any existing information.

DM (Data mode) switches user from analy sis mode to data mode.

LO (Log out) to terminate from the MISS system, returning to the FOCAL environment. 
within each program. And third, commonly used data sets had to be entered separately for each analysis performed, since the output of one program could not be automatically input into another.

These problems were solved by vastly expanding the FCOM function provided in standard LIBRA. As originally programmed, FCOM could store a maximum of five values to be transferred between programs. This number is somewhat inadequate for stand-alone programs, and it certainly precludes the development of an integrated system of programs. The additional storage space needed for FCOM expansion came from our second DF32 disk. At the sacrifice of some operating speed, each user was given a storage space of over 800 values. This is more than enough space for the kinds of instructional computing for which MISS was designed.

Figure 3 is a map of FCOM storage as designed within MISS. Locations of FCOM with negative values are used for storing flags that describe the state of the data during analysis, thus enabling convenient data editing and movement between analysis programs. Positive FCOM locations are used for storing the data sets and for temporary computational space.

As an integrated system, MISS has an entry point that is common to all its programs. This entry program, called MASTER, takes care of the user interaction that is necessary to describe the data (see Table 2 for permissible data formats) and the problem to the system. This frees valuable space within the analysis programs that was previously taken up with data entry.

Program calls are made from MASTER and are decoded by a program named SWITCH, which actually references the called programs. Following the execution of any analysis or editing subroutine, the user is returned to the data mode or analysis mode action commands located within MASTER. This arrangement is economical of space not only in eliminating the wasting of valuable space through repeated data entry but also in enabling a number of programs to share a common subroutine. The matrix inversion program, for example, is used as a subroutine within several other programs.

Most program segments perform more than one function. The particular function of a program is selected by setting FCOM( $\emptyset)$ to a function code that is unique to a program and that is determined initially by the user's request within MASTER. When one of the multipurpose programs is entered, the value of $\operatorname{FCOM}(\emptyset)$ is used to transfer to the appropriate entry point. When control is returned to MASTER following execution of that program segment, the value of $\operatorname{FCOM}(\phi)$ is reset to zero to avoid the propagation of the selected function beyond the original call.

Besides being used for FCOM extension, the disk is also used for the semipermanent storing of data sets, which are accessed by the function FILE. A user may specify within MASTER that his data are to come from a prestored file (these are limited to raw data format). MISS will then copy the selected variables from the file
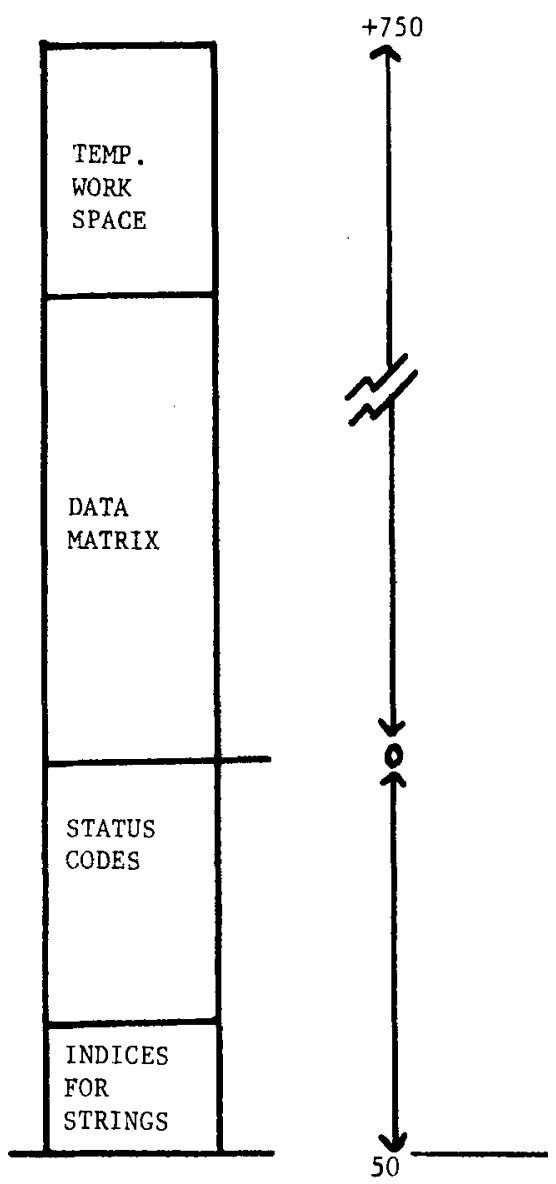

FCOM STORAGE ALLOCATION

Fig. 3. FCOM storage allocation.

in to FCOM so that the analysis can proceed in the same manner as when data are entered from the terminal unit.

\section{HARDWARE ENVIRONMENT}

MISS is currently running on the hardward configuration shown in Fig. 4. This configuration supports the FOCAL/LIBRA operating system. Several substitutions may be made without any FOCAL reprogramming required. Any PDP-8 series computer could be substituted for the PDP-8/L, a PTO 8 multiplexer could be substituted for the $\mathrm{DCO} 2$, and a RFO8 disk could be used instead of a DF32. Any combination of these components would make a system that would run the FOCAL/LIBRA operating system necessary to implement MISS. If a transfer is to be made to a different operating system, three requirements must be met. First, it must have a FOCAL interpreter. FOCAL is the language in which the MISS programs are written. Second, it must have the ability to chain programs. MISS is written in several segments which call each other. Finally, the system must be able to support the disk file access function FCOM. Some candidates for host systems would be the TSS/8 time-sharing system, 


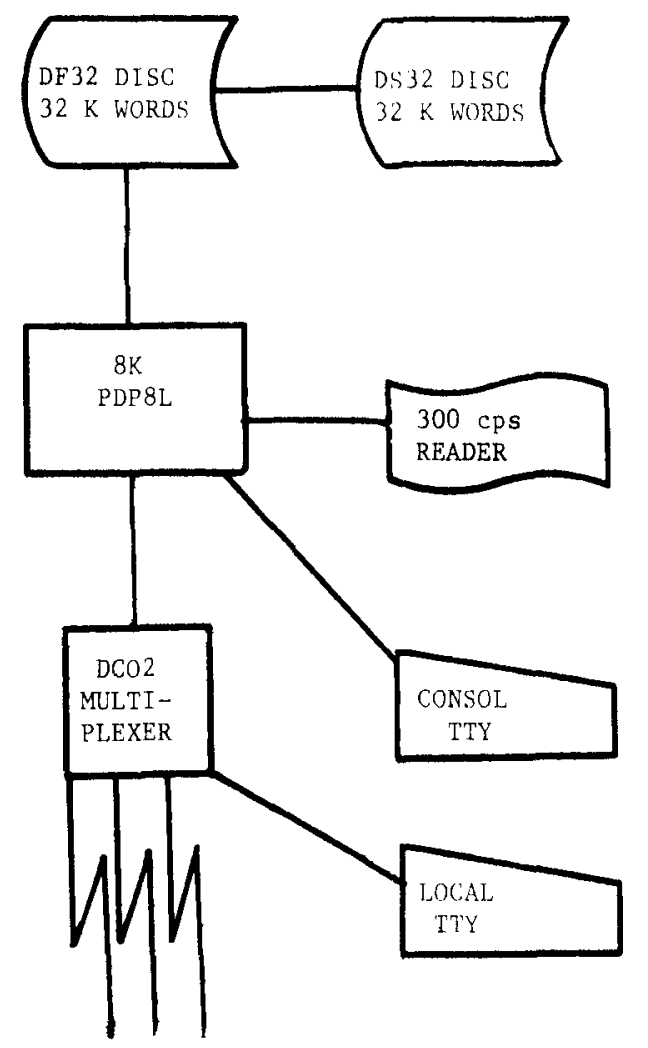

3 DATASETS

Fig. 4. Current configuration for MISS.

the PDP-11 DOS system, and the PDP-10 with the new FOCL/F interpreter.

\section{DEVELOPMENT: EXTENSIONS OF MISS UNDER OS/8}

Since MISS has proven itself as a useful tool, the desire for extensions to its capabilities is strong. The Social Science Research Facilities Center at the University of Minnesota has a PDP-8/E and runs the $\mathrm{OS} / 8$ operating system. This computer configuration, shown in Fig. 5, has several useful peripherals which the time-shared 8L does not have, e.g., an incremental plotter, a graphics display scope, card reader, and large disk. In addition to the useful hardware, the OS/8 operating system is much more standardized and portable than the LIBRA operating system. A special version of FOCAL is being written to take advantage of the graphic devices. Single programs to make histograms, scattergrams, line graphs, and stereoscopic displays have been written. We are now in the process of merging MISS and the special FOCAL to create an extended MISS. In particular, it will take advantage of punched card reading capability and the large disk.

In the process of modifying FOCAL to take advantage of both the hardware and the software of the PDP-8/E computer, a complete rewriting of FOCAL grew into the construction of a language definition (called Formula Language for Interactive Plotting). FLIP is completely backward compatible so that all programs running under FOCAL/LIBRA may be directly transferred. It will, however, enable the following features to be implemented in MISS.

*Data can be entered into MISS from cards. (Most of the data we have is in punched card form.)

*Data files may be very long, since the only limiting factor is the capacity of the disk.

${ }^{*}$ Graphic displays of statistical process will be generated quickly on the scope, and hard copies of the display may be made on the plotter.

FLIP not only makes these extensions possible but alsu improves the performance of MISS. The use of the EAE hardware option speeds up floating point calculations tremendously. Changes in the syntax also allow more efficient coding. Disk access is paged to reduce the number of accesses, and data is stored in three formats to optimize space.

\section{CONCLUSION}

The foregoing discussion of the Minnesota Interactive Statistical System testifies to the viability of on-line minicomputing in an instructional and research context. It is, to our knowledge, unique in being the only fully integrated interactive statistical system presently operating on a minicomputer. (Minicomputer is defined in Anderson \& Gross, 1972.) This approach warrants further attention.

\section{AVAILABILITY}

Copies of the user's manual, The MISS Primer, and

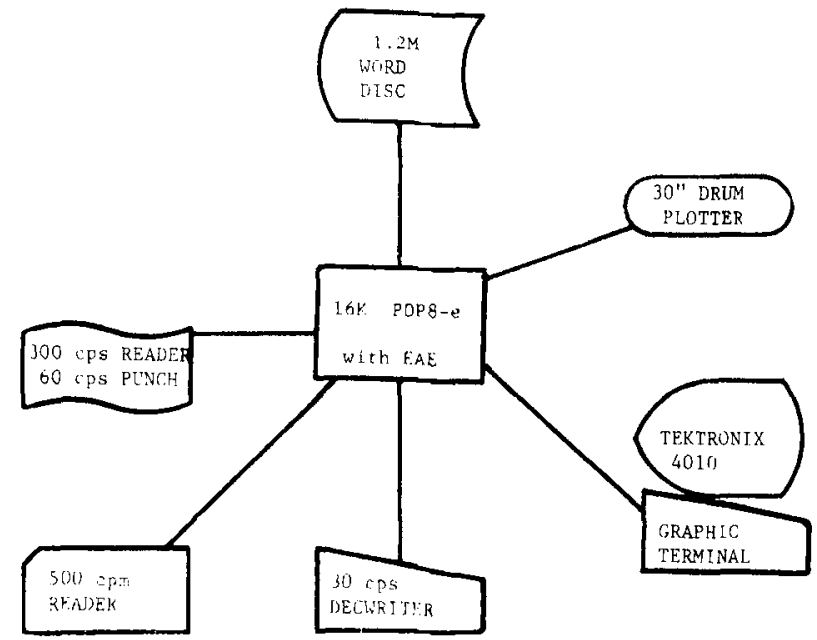

Fig. 5. Graphics-oriented PDP-8/E configuration at Social Sciences Research Facilities Center, University of Minnesota. 
materials for transporting the MISS/LIBRA system are available from James Cleary, Sociology Data Center, Department of Sociology, University of Minnesota, Minneapolis, Minnesota 55455.

\section{REFERENCES}

Anderson, R. E. A survey of application software for social data analysis instruction. Proceedings of a Conference on Computers in the Undergraduate Curricula, Dartmouth College, Hanover, New Hampshire, 1971. Pp. 135-141.

Anderson, R. E., \& Coover, E. R. Wrapping up the package: Critical thoughts on applications software for social data analysis. Computers and the Humanities, 1972, 7, 81-95.

Anderson, R. E., \& Gross, J. Mini-computers in a social science instructional context. Proceedings of the ACM Conference, 1972.

Cleary, J. D. MISS primer. Minneapolis, Minn: Sociology Data
Center, Department of Sociology, University of Minnesota, 1973.

Cline, H. H., \& Meyers, D. Problem-solving computer systems for instruction in sociology. American Sociologist, 1970, 5, 265-270.

\section{NOTES}

1.John Vincent is presently an Assistant Professor of Sociology at the University of Minnesota and is an instructor in the statistics/methods sequence,

2. Due to space limitations, extensive labeling of output is not available on MISS. This limitation is minimized, however, by supplying or constructing code books for the data sets.

3. This limitation on the size of the raw data matrix does not apply to files residing on disk storage. In other words, a large data file might be stored on the disk, but only 700 elements from that file could be analyzed at any one time.

4. In a hardware configuration offering more disk storage area than our hardware system permits, these space limitations of MISS could easily be lifted.

Behavior Research Methods \& Instrumentation

1974, Vol. 6, No. 2, 200-204

\title{
OMNI-SHRIMP: An interpretive language which mimics OMNITAB*
}

\author{
G. SCOTT HARRIS, JAMES M. SWANSON, and JOANNE L. DUERR \\ University of Texas, Austin, Texas 78712
}

\begin{abstract}
A description of OMNITAB, an interpretive language, is provided along with a discussion of its use. The major attributes of SHRIMP (ah interactive mathematical package developed at the University of Texas) are pointed out in detail and its use and relationship to OMNITAB defined. An example is included to show SHRIMP compatibility with OMNITAB.
\end{abstract}

OMNITAB is an interpretive language developed by the National Bureau of Standards for use in statistical and mathematical problems in research. The OMNITAB language is easy to learn because it resembles English, and only a small portion of the language is necessary for the beginner.

Two successful applications of OMNITAB in the classroom have been described in the literature. A paper by Swanson, Harris, and Ledlow (1973) gives a description of an application for teaching undergraduate statistics to students completely unfamiliar with both statistical concepts and the computer. Furthermore, Swanson, Riederer, and Weekly (1973) describe an application in a graduate course for teaching matrix methods in applied statistics.

\section{HISTORICAL BACKGROUND}

The University of Texas has a long-standing involvement in academic computing. This involvement led to the development of a large academic Computation Center, independent of administrative computing, comprised of a Control Data 6400 and a Control Data 6600 together with Texas's own dual operating system. Appended to this are two high-speed remote terminals, 15 medium-speed remote terminals, and 64 Teletype

* Reprints may be obtained from G. Scott Harris, Computation Center, University of Texas, Austin, Texas 78712 . lines, soon to be doubled.

There is a good reason for mentioning these figures. They indicate a growing trend in a university such as Texas (with 40,000 students) to make available a new form of computing: time-shared interactive instruction. The OMNITAB and SHRIMP systems illustrate the use of facilities such as these in a new way.

Previously, academic computing has been heavily weighted toward numerical methods in mathematics and the physical sciences. In recent years, however, academic computing has been developing interactive tutorial systems, such as CLIC at Texas, for student drill work. Now there is another use of the computer which lies between the page-flipping drill sessions and the mastery of a complex computer language.

An example of this kind of application is the OMNITAB system. Originally used for research when developed by the National Bureau of Standards, it has a number of features which fill this gap. First, it resembles English and is easy for the beginner to master with the positive feedback of early success. Second, it is a true procedural language, placing the power of the computer under the user's control (see Hogben, Peavy, \& Varner, 1971). Third, when using the modifications of the University of Texas, a user receives immediate feedback on his every directive and even on instructions given by a professor (see Harris, 1971).

These features have made OMNITAB extremely 